\title{
Supporting information \\ Langevin Dynamics Simulations of the Exchange of Complex Coacervate Core Micelles: The Role of Nonelectrostatic Attraction and Polyelectrolyte Length
}

\author{
Inge Bos $^{1}$ and Joris Sprakel ${ }^{* 1}$ \\ ${ }^{1}$ Physical Chemistry and Soft Matter, Wageningen University \& Research, Stippeneng 4, \\ 6708 WE, Wageningen, The Netherlands
}

\section{SI 1 Interaction potentials}

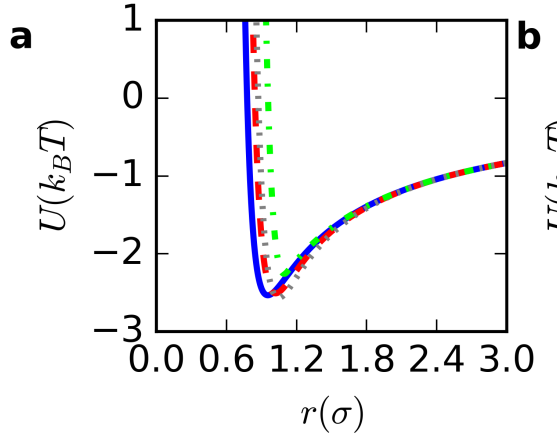

- Coulomb + LJ : $\varepsilon_{L J}=0.05$

- Coulomb + LJ : $\varepsilon_{L J}=0.15$

$\cdots \quad$ Coulomb + LJ $: \varepsilon_{L J}=0.25$

-. Coulomb + WCA

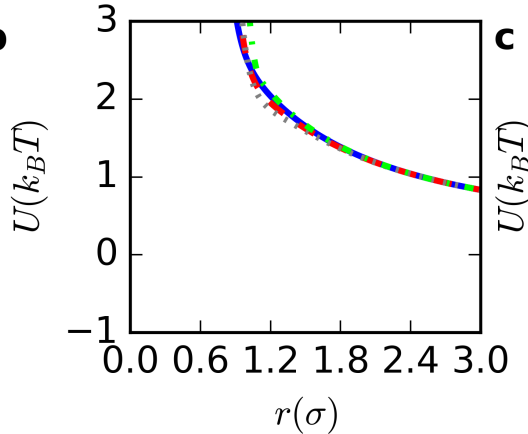

— Coulomb + LJ : $\varepsilon_{L J}=0.05$

- Coulomb + LJ : $\varepsilon_{L J}=0.15$

,... Coulomb + LJ $: \varepsilon_{L J}=0.25$

- Coulomb + WCA

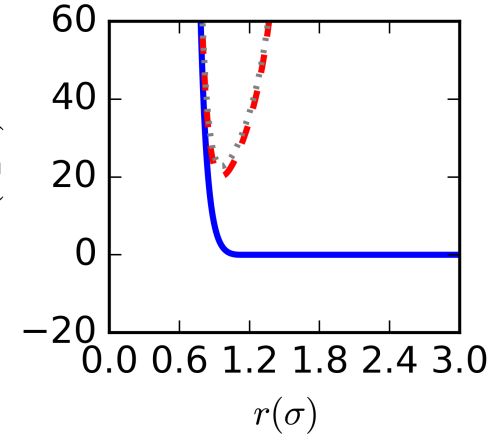

- WCA

- FENE + WCA

‥ FENE + WCA + Coulomb

Figure SI 1.1: The interaction potentials used in the Langevin dynamics simulations. a) Coulomb potential for oppositely charged particles combined with a Lennard-Jones (LJ) potential or a Weeks-Chandler-Anders (WCA) potential b) Coulomb potential for equally charged particles combined with a Lennard-Jones (LJ) potential or a Weeks-Chandler-Anders (WCA) potential c) Weeks-Chandler-Anders (WCA) potential, a finitely extensible nonlinear elastic (FENE) potential combined with a WCA potential and a finitely extensible nonlinear elastic (FENE) potential combined with a WCA potential and a Coulomb potential.

${ }^{*}$ Corresponding author: joris.sprakel@wur.nl 


\section{SI 2 C3M formation}
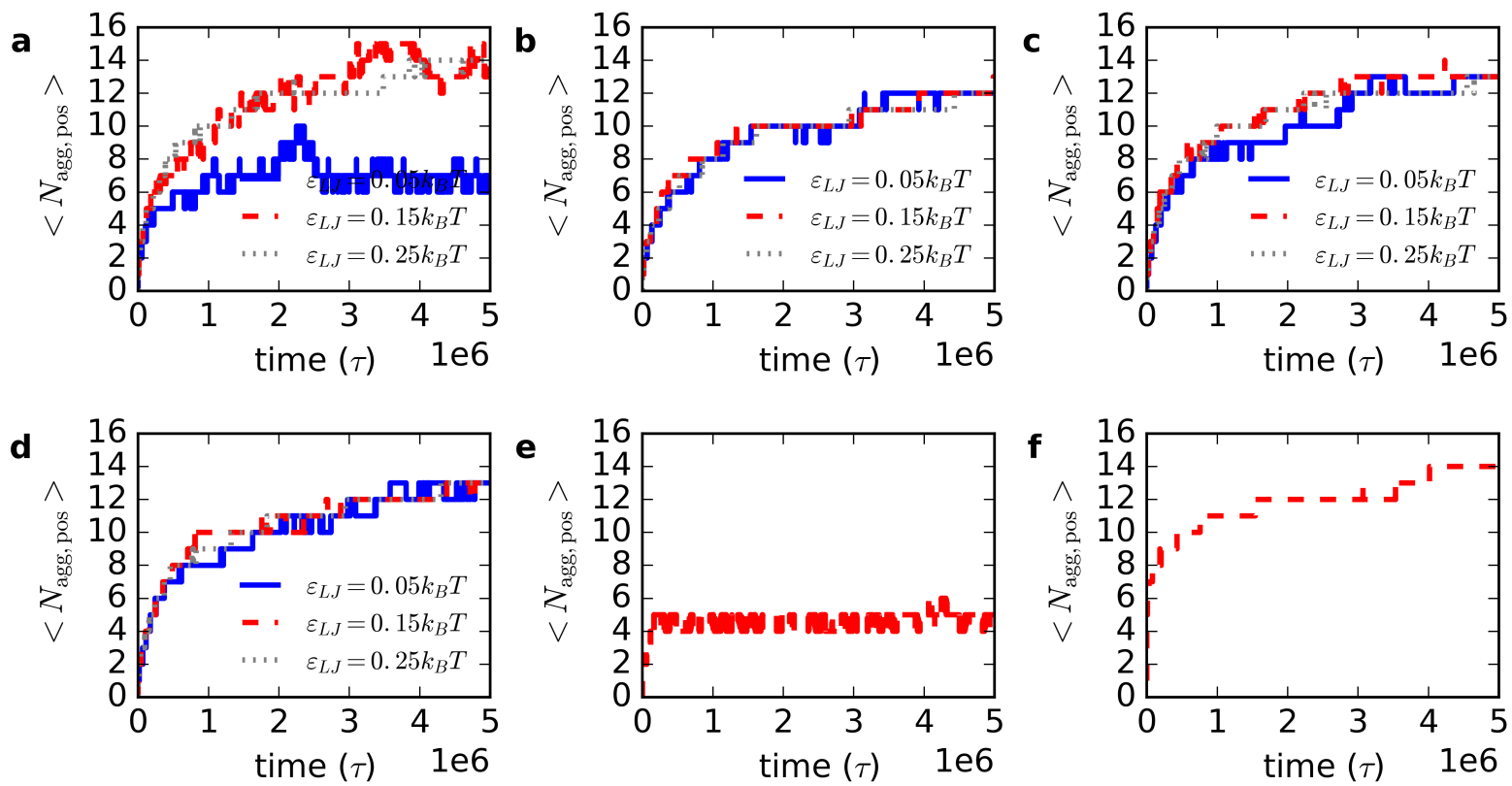

Figure SI 2.1: The increase of the average micelle size, expressed as the average aggregation number of positive blocks per micelle $N_{\text {agg,pos }}$ in time for a) $N_{\text {pos }}=N_{\text {neg }}=20$ and $N_{\text {neu }}=50$, b) $N_{\text {pos }}=N_{\text {neg }}=30$ and $N_{\text {neu }}=75$, c) $N_{\text {pos }}=20, N_{\text {neg }}=15$ and $N_{\text {neu }}=50$, d) $N_{\text {pos }}=20, N_{\text {neg }}=25$ and $N_{\text {neu }}=50$, e) $N_{\text {pos }}=20$, $N_{\text {neg }}=20$ and $N_{\text {neu }}=50$ and only nonelectrostatic attraction between the positive polyelectrolytes and f) $N_{\text {pos }}=20, N_{\text {neg }}=150, N_{\text {neu }}=50$ and $\varepsilon_{L J}=0.15 k_{B} T$. 

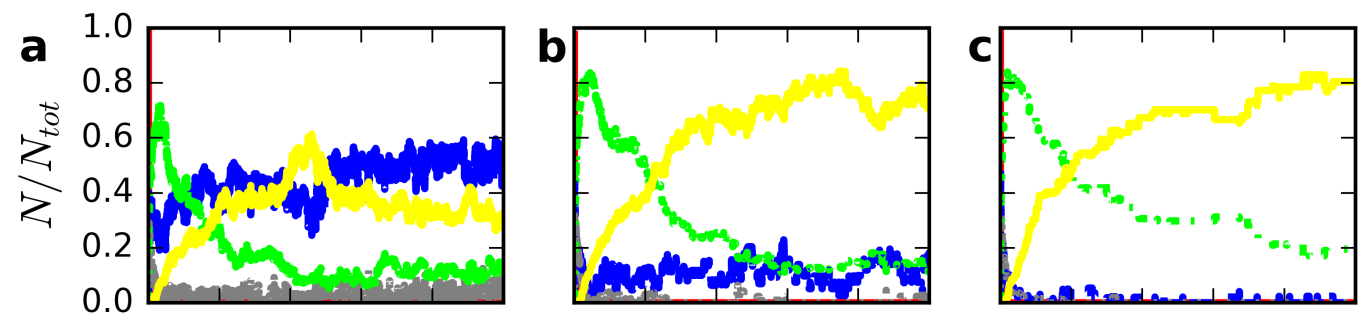

$$
\begin{array}{ll}
- & N_{\text {agg }}=1 \\
-\because & N_{\text {agg }}=2 \\
\cdots \cdots & N_{\text {agg }}=3-4 \\
\because \cdots & N_{\text {agg }}=5-20 \\
= & N_{\text {agg }}>20
\end{array}
$$
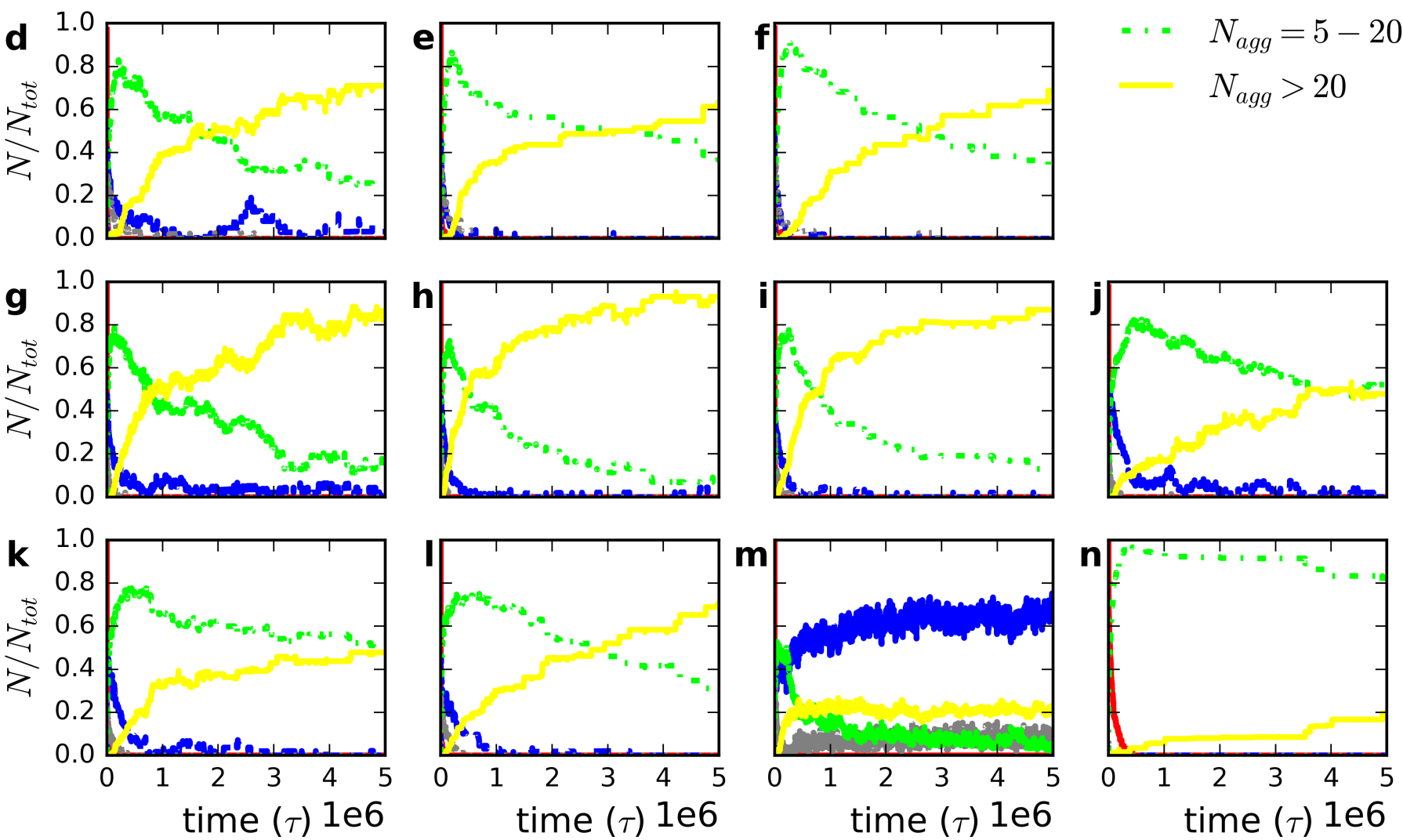

Figure SI 2.2: Time development of the number of clusters $N$ with a certain aggregation number $N_{a g g}$ compared to the total number of clusters $N_{t o t}$ at this time step for

a,b,c) $N_{\text {pos }}=30, N_{n e g}=20$ and $N_{n e u}=50$ and $\varepsilon_{L J}=0.05$ (a), $\varepsilon_{L J}=0.15$ (b) and $\varepsilon_{L J}=0.25$ (c), d,e,f) $N_{\text {pos }}=30, N_{\text {neg }}=30$ and $N_{\text {neu }}=75$ and $\varepsilon_{L J}=0.05(\mathrm{~d}), \varepsilon_{L J}=0.15$ (e) and $\varepsilon_{L J}=0.25$ (f), $\mathrm{g}, \mathrm{h}, \mathrm{i}) N_{\text {pos }}=20, N_{\text {neg }}=15$ and $N_{\text {neu }}=50$ and $\varepsilon_{L J}=0.05(\mathrm{~g}), \varepsilon_{L J}=0.15(\mathrm{~h})$ and $\varepsilon_{L J}=0.25$ (i), $\mathrm{j}, \mathrm{k}, \mathrm{l}) N_{\text {pos }}=20, N_{\text {neg }}=25$ and $N_{\text {neu }}=50$ and $\varepsilon_{L J}=0.05(\mathrm{j}), \varepsilon_{L J}=0.15(\mathrm{k})$ and $\varepsilon_{L J}=0.25(\mathrm{l})$ m) $N_{\text {pos }}=20, N_{\text {neg }}=20$ and $N_{\text {neu }}=50$ and only nonelectrostatic attraction between the positive polyelectrolytes and

n) $N_{\text {pos }}=20, N_{\text {neg }}=150, N_{\text {neu }}=50$ and $\varepsilon_{L J}=0.15 k_{B} T$. 

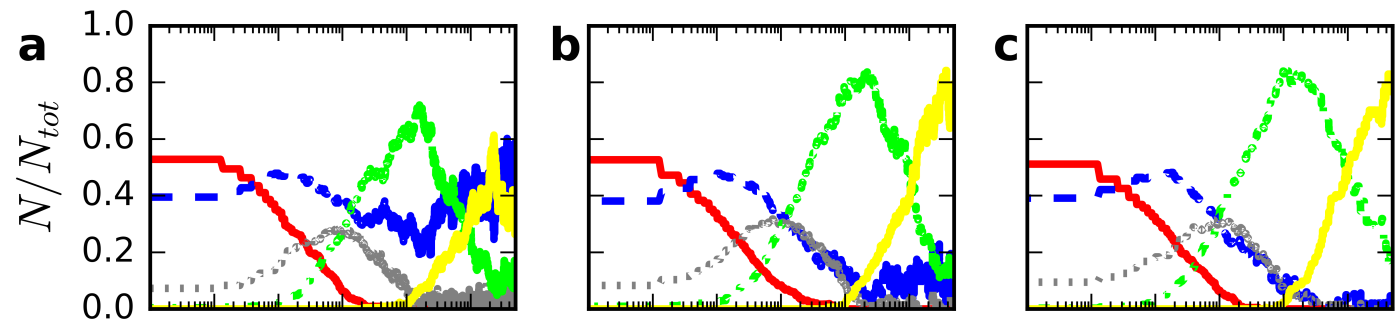

$-\quad N_{a g g}=1$

- $\quad N_{a g g}=2$

$N_{\text {agg }}=3-4$
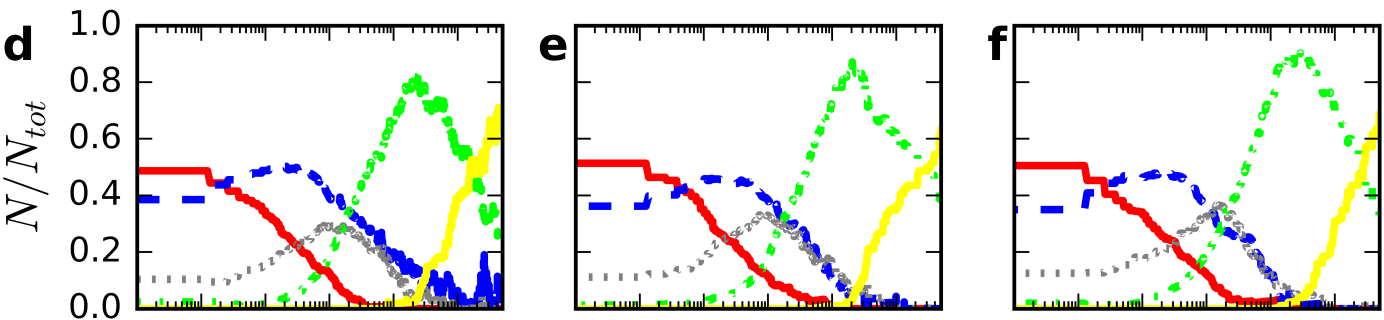

$N_{a g g}=5-20$

$N_{a g g}>20$
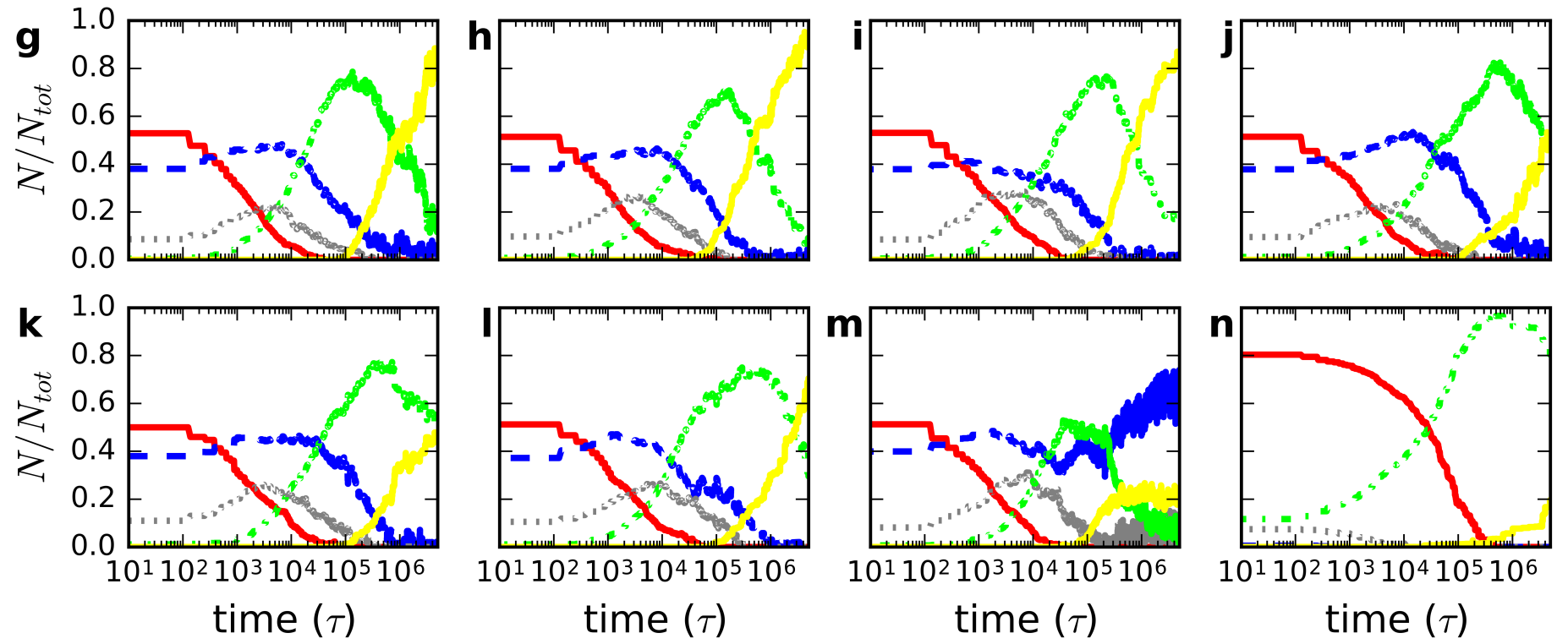

Figure SI 2.3: The same data as in Fig. SI 2.2, but now plotted with a logarithmic time axis: Time development of the number of aggregates $N$ with a certain aggregation number $N_{\text {agg }}$ compared to the total number of aggregates $N_{\text {tot }}$ at this time step for

a,b,c) $N_{\text {pos }}=30, N_{n e g}=20$ and $N_{\text {neu }}=50$ and $\varepsilon_{L J}=0.05$ (a), $\varepsilon_{L J}=0.15$ (b) and $\varepsilon_{L J}=0.25$ (c), d,e,f) $N_{\text {pos }}=30, N_{\text {neg }}=30$ and $N_{\text {neu }}=75$ and $\varepsilon_{L J}=0.05$ (d), $\varepsilon_{L J}=0.15$ (e) and $\varepsilon_{L J}=0.25$ (f), $\mathrm{g}, \mathrm{h}, \mathrm{i}) N_{\text {pos }}=20, N_{\text {neg }}=15$ and $N_{\text {neu }}=50$ and $\varepsilon_{L J}=0.05(\mathrm{~g}), \varepsilon_{L J}=0.15(\mathrm{~h})$ and $\varepsilon_{L J}=0.25$ (i), $\mathrm{j}, \mathrm{k}, \mathrm{l}) N_{\text {pos }}=20, N_{n e g}=25$ and $N_{\text {neu }}=50$ and $\varepsilon_{L J}=0.05(\mathrm{j}), \varepsilon_{L J}=0.15(\mathrm{k})$ and $\varepsilon_{L J}=0.25(\mathrm{l})$ m) $N_{\text {pos }}=20, N_{n e g}=20$ and $N_{n e u}=50$ and only nonelectrostatic attraction between the positive polyelectrolytes and

n) $N_{\text {pos }}=20, N_{\text {neg }}=150, N_{\text {neu }}=50$ and $\varepsilon_{L J}=0.15 k_{B} T$. 
a

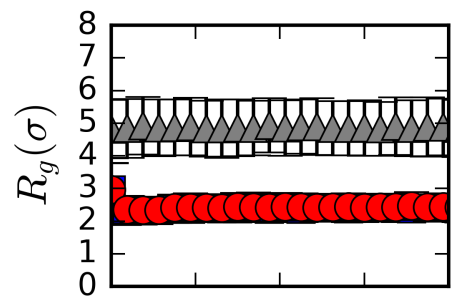

d

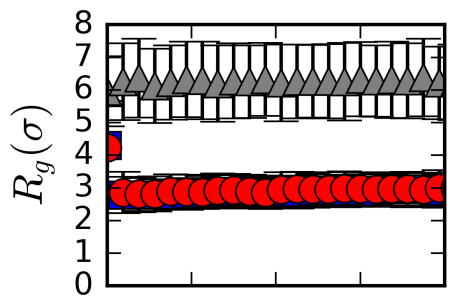

g

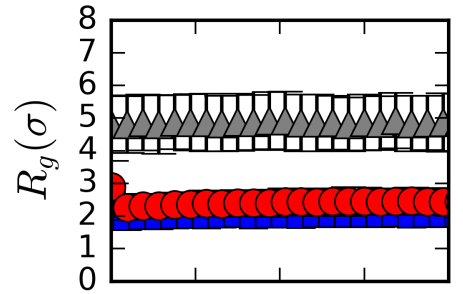

$\mathbf{k}$

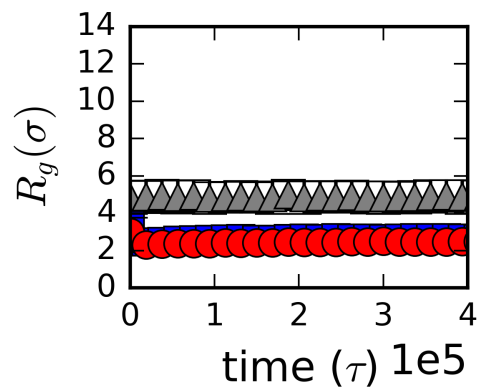

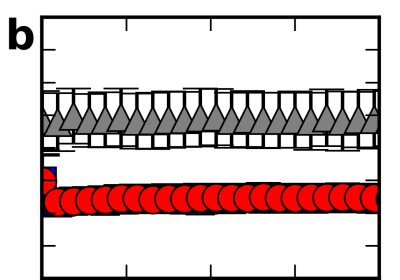
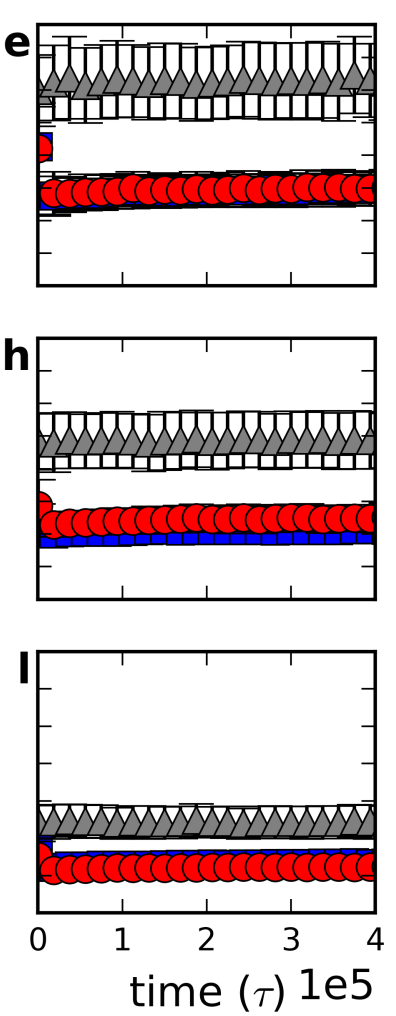
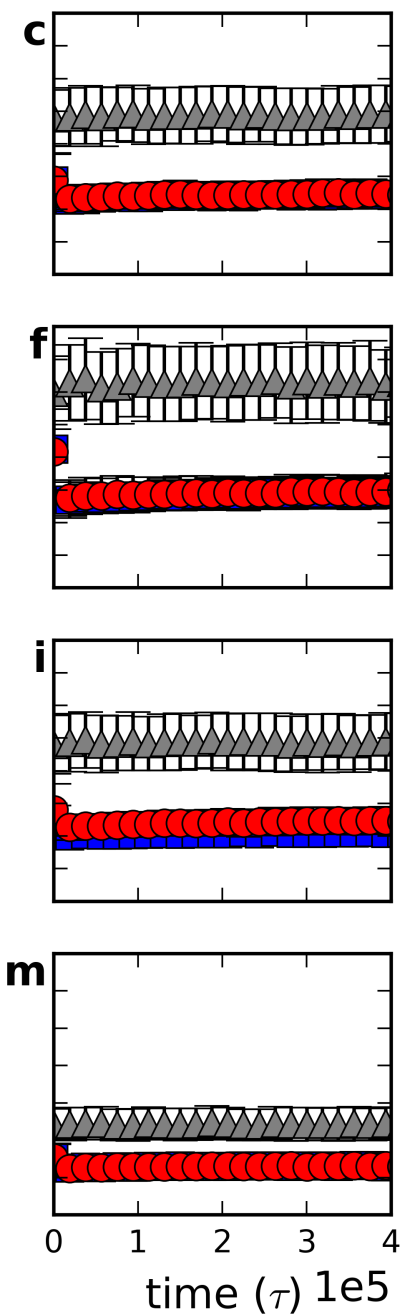

negative polymer positive block neutral block
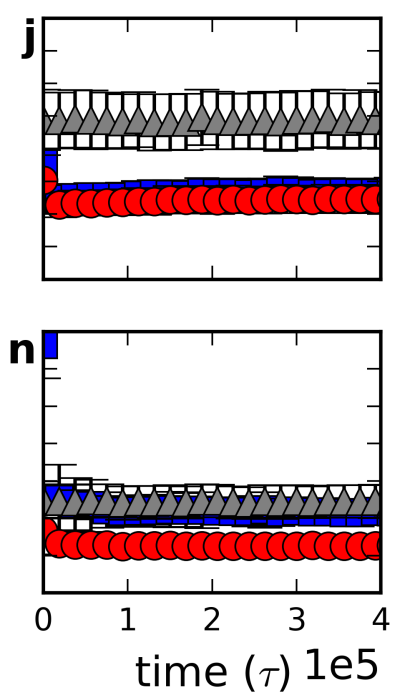

Figure SI 2.4: Early time development of the average radius of gyration $R_{g}$ of the negative polymers, positive blocks and neutral blocks for

a,b,c) $N_{\text {pos }}=30, N_{\text {neg }}=20$ and $N_{\text {neu }}=50$ and $\varepsilon_{L J}=0.05$ (a), $\varepsilon_{L J}=0.15$ (b) and $\varepsilon_{L J}=0.25$ (c), d,e,f) $N_{\text {pos }}=30, N_{\text {neg }}=30$ and $N_{\text {neu }}=75$ and $\varepsilon_{L J}=0.05(\mathrm{~d}), \varepsilon_{L J}=0.15$ (e) and $\varepsilon_{L J}=0.25$ (f), $\mathrm{g}, \mathrm{h}, \mathrm{i}) N_{\text {pos }}=20, N_{\text {neg }}=15$ and $N_{\text {neu }}=50$ and $\varepsilon_{L J}=0.05(\mathrm{~g}), \varepsilon_{L J}=0.15(\mathrm{~h})$ and $\varepsilon_{L J}=0.25$ (i), $\mathrm{j}, \mathrm{k}, \mathrm{l}) N_{\text {pos }}=20, N_{\text {neg }}=25$ and $N_{\text {neu }}=50$ and $\varepsilon_{L J}=0.05(\mathrm{j}), \varepsilon_{L J}=0.15(\mathrm{k})$ and $\varepsilon_{L J}=0.25(\mathrm{l})$ m) $N_{\text {pos }}=20, N_{\text {neg }}=20$ and $N_{\text {neu }}=50$ and only nonelectrostatic attraction between the positive polyelectrolytes and

n) $N_{\text {pos }}=20, N_{\text {neg }}=150, N_{\text {neu }}=50$ and $\varepsilon_{L J}=0.15 k_{B} T$. 

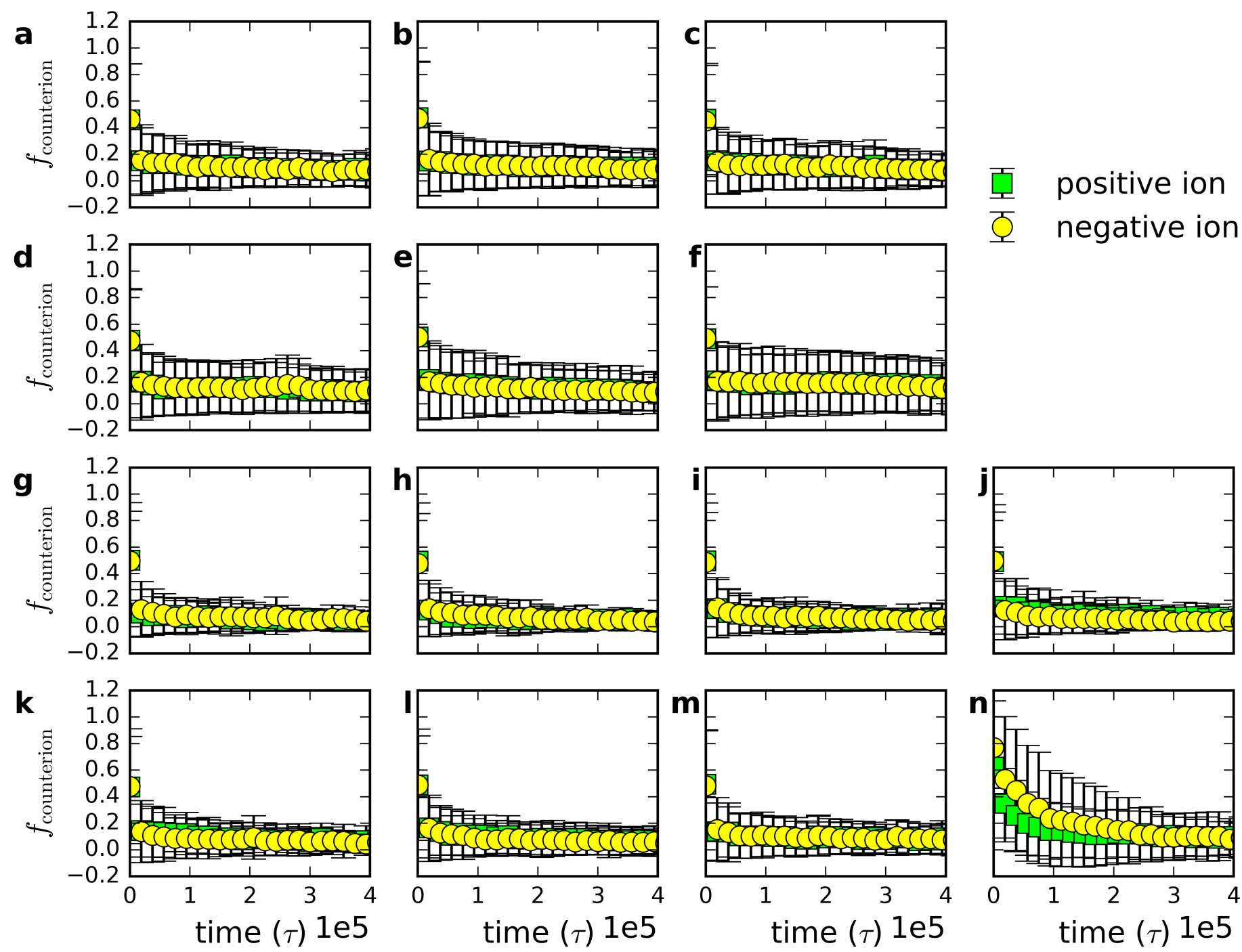

Figure SI 2.5: Early time development of the average fraction of bound counter ions $f_{\text {counter ion. Here the }}$ fraction of bound counter ions is calculated by the number of counter ions present within a distance $2.5 \sigma$ from the oppositely charged monomers of an cluster (single polymer or complex) divided by the total number of oppositely charged monomers in this cluster. Data points are averages over all clusters present at a time step. The data points are obtained from simulations with

a,b,c) $N_{\text {pos }}=30, N_{n e g}=20$ and $N_{\text {neu }}=50$ and $\varepsilon_{L J}=0.05$ (a), $\varepsilon_{L J}=0.15$ (b) and $\varepsilon_{L J}=0.25$ (c), d,e,f) $N_{\text {pos }}=30, N_{\text {neg }}=30$ and $N_{\text {neu }}=75$ and $\varepsilon_{L J}=0.05(\mathrm{~d}), \varepsilon_{L J}=0.15$ (e) and $\varepsilon_{L J}=0.25$ (f), $\mathrm{g}, \mathrm{h}, \mathrm{i}) N_{\text {pos }}=20, N_{\text {neg }}=15$ and $N_{\text {neu }}=50$ and $\varepsilon_{L J}=0.05(\mathrm{~g}), \varepsilon_{L J}=0.15(\mathrm{~h})$ and $\varepsilon_{L J}=0.25(\mathrm{i})$, $\mathrm{j}, \mathrm{k}, \mathrm{l}) N_{\text {pos }}=20, N_{\text {neg }}=25$ and $N_{\text {neu }}=50$ and $\varepsilon_{L J}=0.05(\mathrm{j}), \varepsilon_{L J}=0.15(\mathrm{k})$ and $\varepsilon_{L J}=0.25(\mathrm{l})$ m) $N_{\text {pos }}=20, N_{\text {neg }}=20$ and $N_{\text {neu }}=50$ and only nonelectrostatic attraction between the positive polyelectrolytes and

n) $N_{\text {pos }}=20, N_{\text {neg }}=150, N_{\text {neu }}=50$ and $\varepsilon_{L J}=0.15 k_{B} T$. 

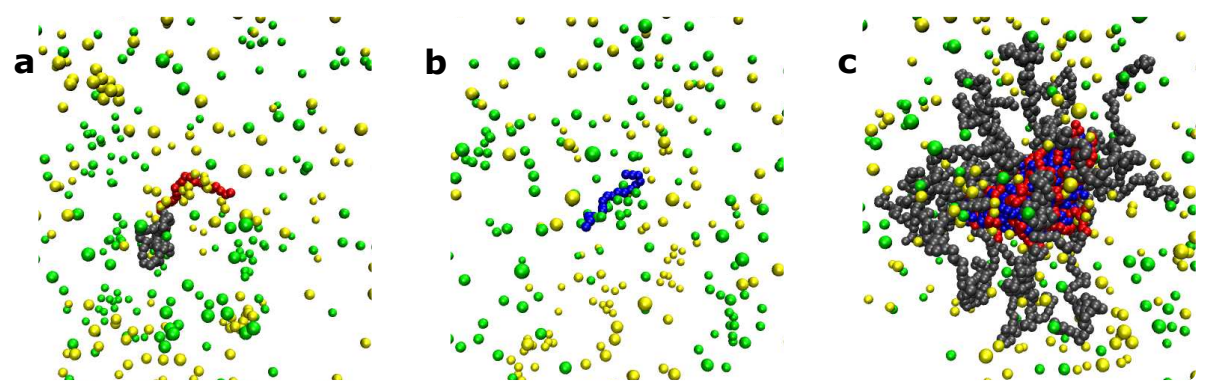

Figure SI 2.6: Example of the ion distribution around a) a diblock, b) a negative polymer and c) a C3M. Images are snapshots from the simulations at $63 \tau(\mathrm{a}, \mathrm{b})$ and $5 \times 10^{6} \tau(\mathrm{c})$. Negative homopolymers are shown in blue, the positive block in red, the neutral block in gray, the positive ions in green and the negative ions in yellow.

\section{SI 3 C3M configurations}
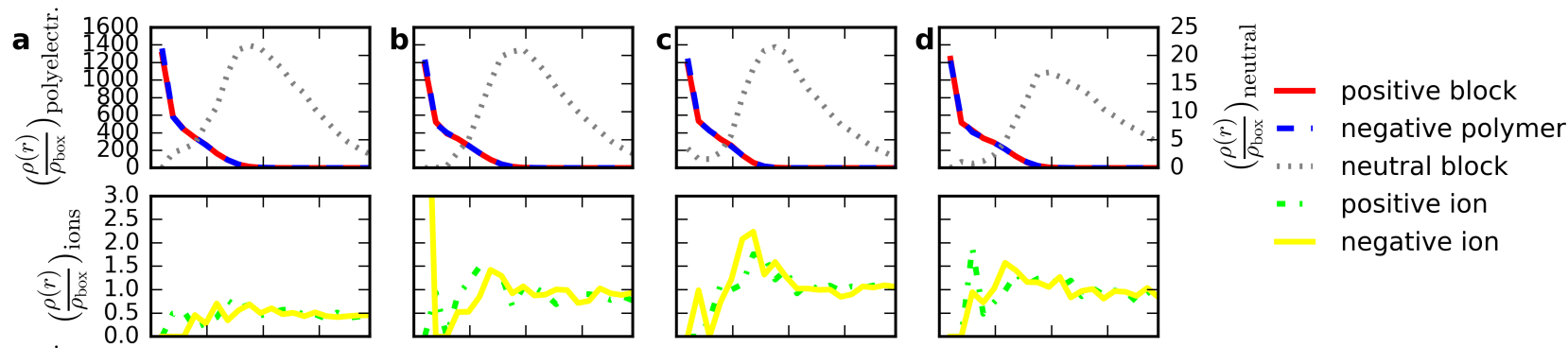

positive ion
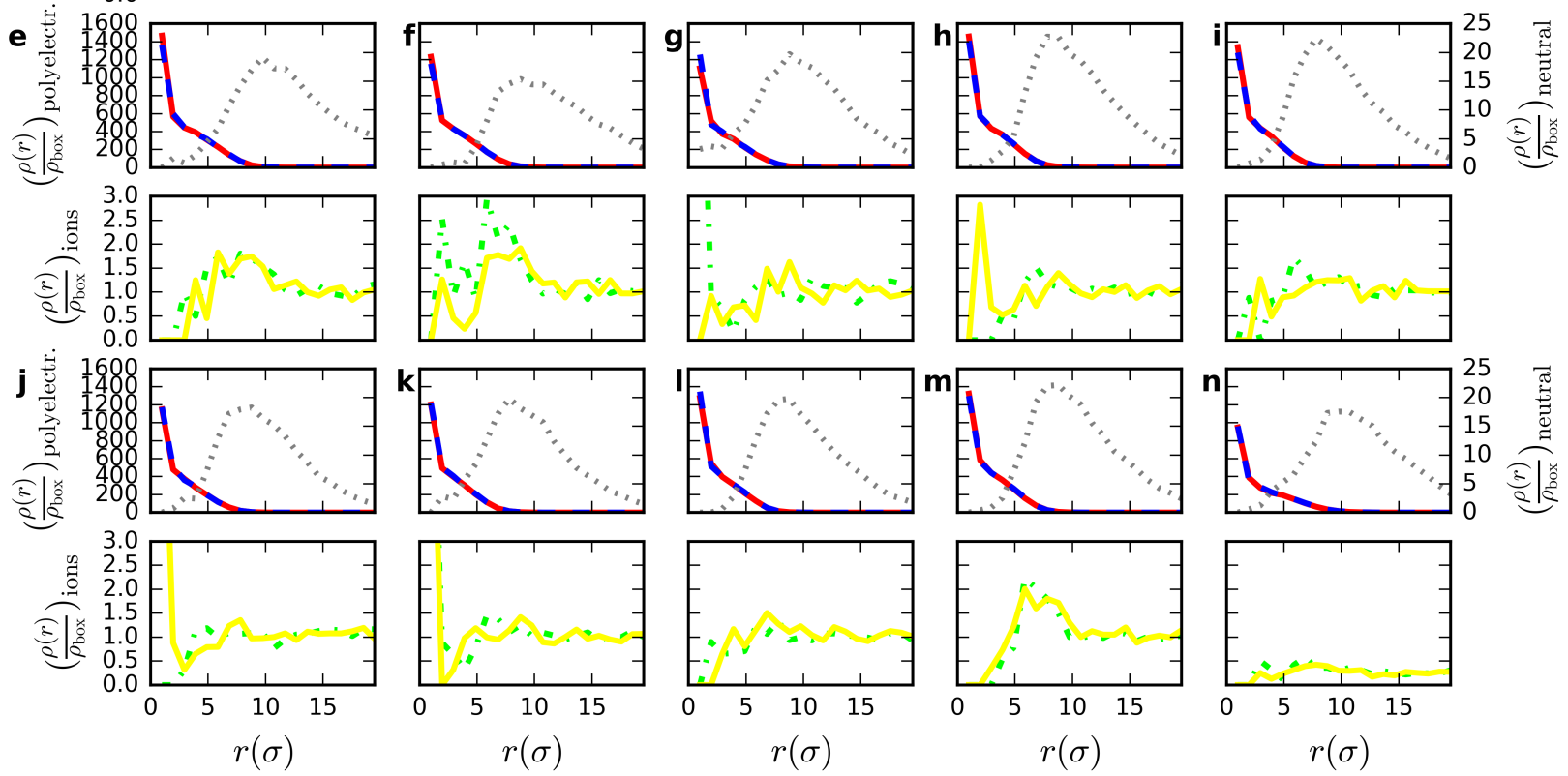

Figure SI 3.1: Normalized density profiles as function of the distance $r$ from the mass centre of the micelle. The density profiles are averaged over all micelles present at the end of the simulation for a,b,c) $N_{\text {pos }}=30, N_{n e g}=20$ and $N_{\text {neu }}=50$ and $\varepsilon_{L J}=0.05$ (a), $\varepsilon_{L J}=0.15$ (b) and $\varepsilon_{L J}=0.25$ (c), d,e,f) $N_{\text {pos }}=30, N_{n e g}=30$ and $N_{\text {neu }}=75$ and $\varepsilon_{L J}=0.05(\mathrm{~d}), \varepsilon_{L J}=0.15$ (e) and $\varepsilon_{L J}=0.25$ (f), g,h,i) $N_{\text {pos }}=20, N_{\text {neg }}=15$ and $N_{\text {neu }}=50$ and $\varepsilon_{L J}=0.05(\mathrm{~g}), \varepsilon_{L J}=0.15(\mathrm{~h})$ and $\varepsilon_{L J}=0.25$ (i), $\mathrm{j}, \mathrm{k}, \mathrm{l}) N_{\text {pos }}=20, N_{\text {neg }}=25$ and $N_{\text {neu }}=50$ and $\varepsilon_{L J}=0.05(\mathrm{j}), \varepsilon_{L J}=0.15(\mathrm{k})$ and $\varepsilon_{L J}=0.25(\mathrm{l})$ m) $N_{\text {pos }}=20, N_{\text {neg }}=20$ and $N_{\text {neu }}=50$ and only nonelectrostatic attraction between the positive polyelectrolytes and

n) $N_{\text {pos }}=20, N_{\text {neg }}=150, N_{\text {neu }}=50$ and $\varepsilon_{L J}=0.15 k_{B} T$. 

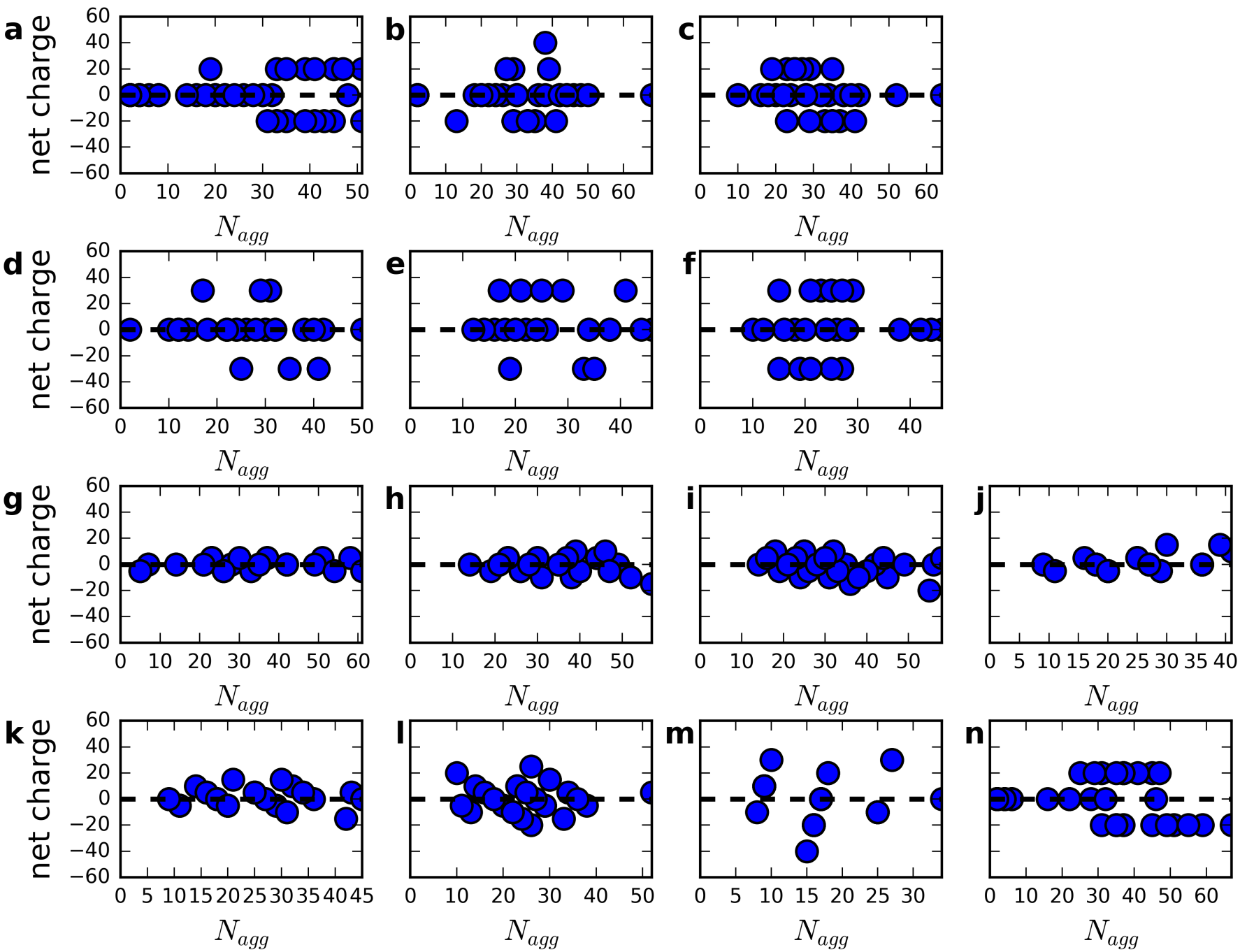

Figure SI 3.2: Net micelle charge versus micelle aggregation number for all micelles present at the end of the simulation for

a,b,c) $N_{\text {pos }}=30, N_{\text {neg }}=20$ and $N_{\text {neu }}=50$ and $\varepsilon_{L J}=0.05$ (a), $\varepsilon_{L J}=0.15$ (b) and $\varepsilon_{L J}=0.25$ (c),

d,e,f) $N_{\text {pos }}=30, N_{\text {neg }}=30$ and $N_{\text {neu }}=75$ and $\varepsilon_{L J}=0.05(\mathrm{~d}), \varepsilon_{L J}=0.15$ (e) and $\varepsilon_{L J}=0.25$ (f),

$\mathrm{g}, \mathrm{h}, \mathrm{i}) N_{\text {pos }}=20, N_{\text {neg }}=15$ and $N_{\text {neu }}=50$ and $\varepsilon_{L J}=0.05(\mathrm{~g}), \varepsilon_{L J}=0.15(\mathrm{~h})$ and $\varepsilon_{L J}=0.25$ (i),

$\mathrm{j}, \mathrm{k}, \mathrm{l}) N_{\text {pos }}=20, N_{\text {neg }}=25$ and $N_{\text {neu }}=50$ and $\varepsilon_{L J}=0.05(\mathrm{j}), \varepsilon_{L J}=0.15(\mathrm{k})$ and $\varepsilon_{L J}=0.25(\mathrm{l})$

m) $N_{\text {pos }}=20, N_{\text {neg }}=20$ and $N_{\text {neu }}=50$ and only nonelectrostatic attraction between the positive polyelectrolytes and

n) $N_{\text {pos }}=20, N_{\text {neg }}=150, N_{\text {neu }}=50$ and $\varepsilon_{L J}=0.15 k_{B} T$. 


\section{SI 4 No electrostatic attraction}
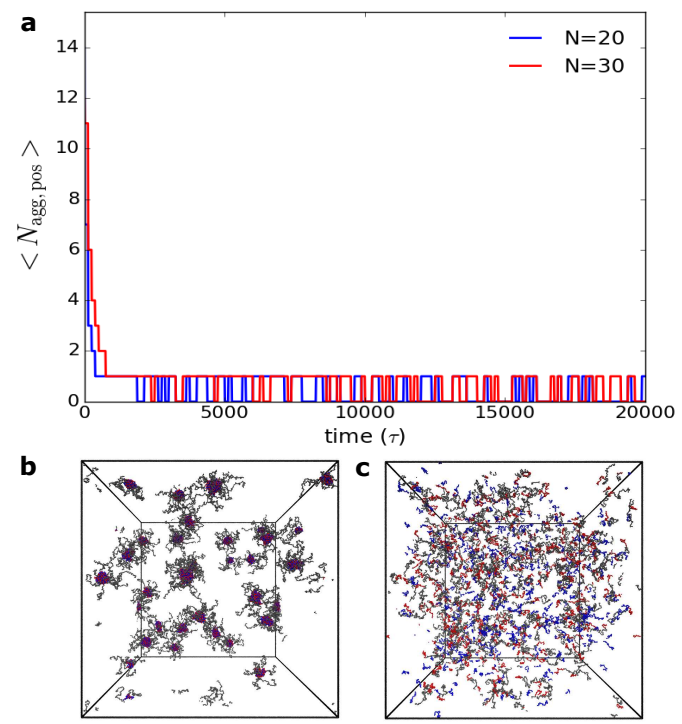

Figure SI 4.1: C3Ms fall apart when the electrostatic attraction is turned off as exemplified here for the end configurations of the simulations with the strongest nonelectrostatic attraction used in this study $\left(\varepsilon_{\mathrm{LJ}}=0.25 k_{B} T\right)$. a) Average aggregation number as function of time after turning off the electrostatic attraction for $N_{n e g}=N_{\text {pos }}=20$ and $N_{\text {neu }}=50$ and for $N_{n e g}=N_{\text {pos }}=30$ and $N_{\text {neu }}=75$. b) Configuration before electrostatic attraction is turned off $($ time $=0 \tau)$ for $N_{\text {neg }}=N_{\text {pos }}=30$ and $N_{\text {neu }}=75$. c) Configuration after turning off the electrostatic attraction (time $\left.=1.25 \times 10^{4} \tau\right)$ for $N_{\text {neg }}=N_{\text {pos }}=30$ and $N_{\text {neu }}=75$.

\section{SI 5 Merge and split events on a logarithmic time axis}
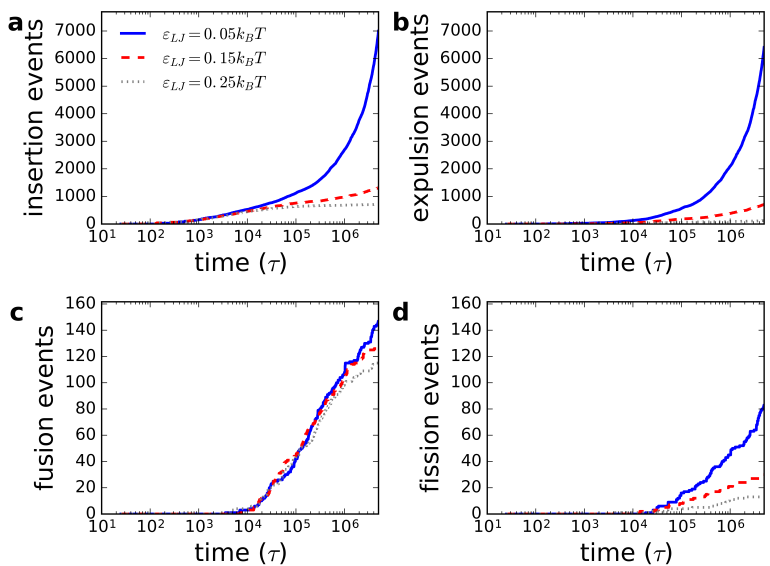

Figure SI 5.2: Cumulative number of insertion (a), expulsion (b), fusion (c) and fission (d) events for a nonelectrostatic attraction strength between the polyelectrolytes of $\varepsilon_{\mathrm{LJ}}=0.05 k_{B} T, \varepsilon_{\mathrm{LJ}}=0.15 k_{B} T$ and $\varepsilon_{\mathrm{LJ}}=0.25 k_{B} T$. In all cases, $N_{\text {neg }}=20, N_{\text {pos }}=20$ and $N_{\text {neu }}=50$. 


\section{SI 6 Mechanisms of C3M growth}
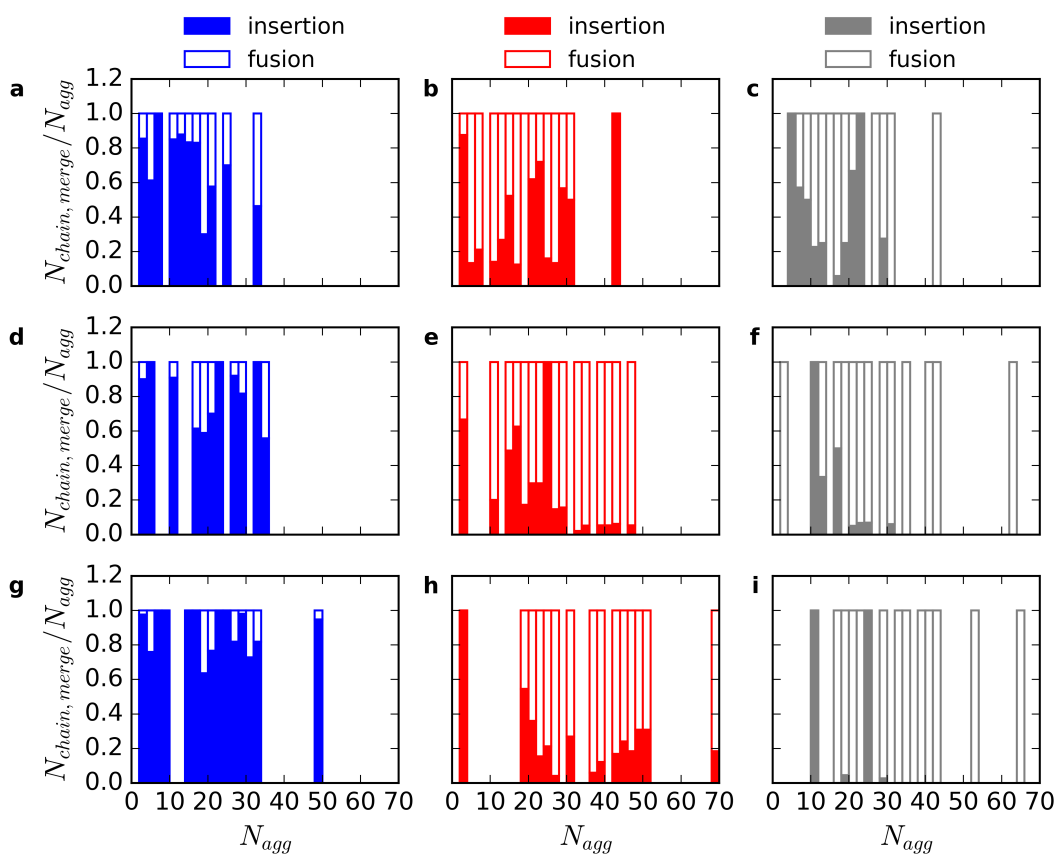

Figure SI 6.1: The fraction of chains coming from insertion and fusion as function of micelle aggregation number at the time points $5 \times 10^{5} \tau$ (a,b,c), $2 \times 10^{6} \tau$ (d,e,f) and $5 \times 10^{6} \tau(\mathrm{g}, \mathrm{h}, \mathrm{i})$ for $N_{\text {pos }}=20, N_{\text {neg }}=20$ and $N_{\text {neu }}=50$ with $\varepsilon_{L J}=0.05 k_{B} T(\mathrm{a}, \mathrm{d}, \mathrm{g}), \varepsilon_{L J}=0.15 k_{B} T(\mathrm{~b}, \mathrm{e}, \mathrm{h})$ and $\varepsilon_{L J}=0.25 k_{B} T$ (c,f,i). The analysis is based on the last merging event that a chain was involved in. A chain is considered to be involved in insertion when it is part of a merging complex with a size smaller than 5. For fusion all chains of both merging complexes are considered to be involved in the fusion.
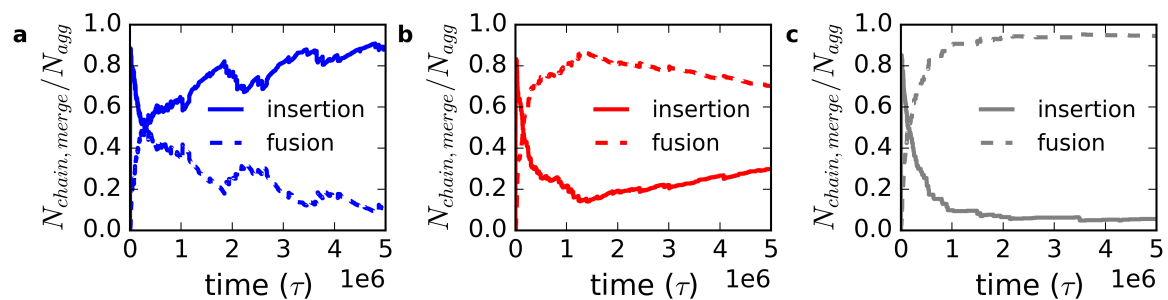

Figure SI 6.2: The fraction of chains coming from insertion and fusion as function of time for $N_{\text {pos }}=20, N_{\text {neg }}=20$ and $N_{\text {neu }}=50$ with a) $\varepsilon_{L J}=0.05 k_{B} T$, b) $\varepsilon_{L J}=0.15 k_{B} T$ and c) $\varepsilon_{L J}=0.25 k_{B} T$.
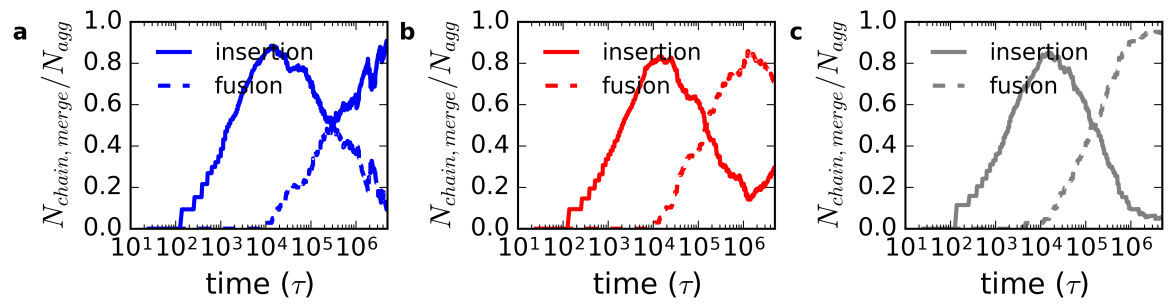

Figure SI 6.3: The same data as in Fig. SI 6.2 but now plotted on a logarithmic time axis: The fraction of chains coming from insertion and fusion as function of time on a logarithmic scale for $N_{\text {pos }}=20, N_{\text {neg }}=20$ and $N_{\text {neu }}=50$ with a) $\varepsilon_{L J}=0.05 k_{B} T$, b) $\varepsilon_{L J}=0.15 k_{B} T$ and c) $\varepsilon_{L J}=0.25 k_{B} T$. Note that when the sum of the insertion and fusion fraction is smaller than 1 this means that some of the chains have not been involved in a merging event yet. 


\section{SI 7 Dimer configurations}
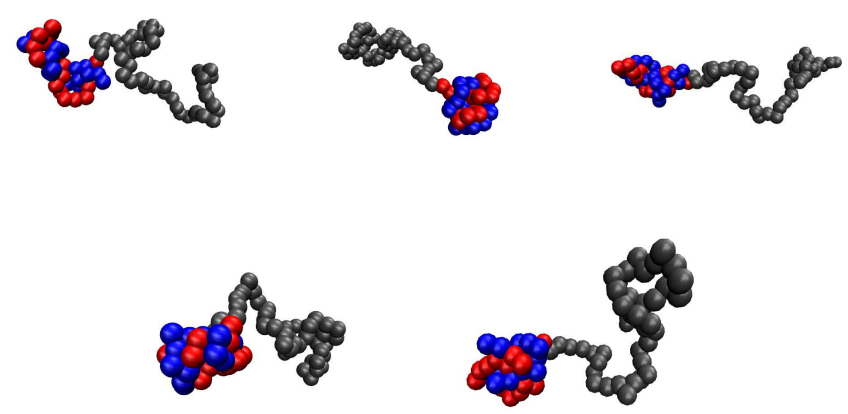

Figure SI 7.1: Examples of free dimer configurations in the last simulation step for $N_{\text {neg }}=20, N_{\text {pos }}=20$, $N_{\text {neu }}=50$ and $\varepsilon_{\mathrm{LJ}}=0.15 k_{B} T$.

SI 8 Split events corrected for the number of polymers involved

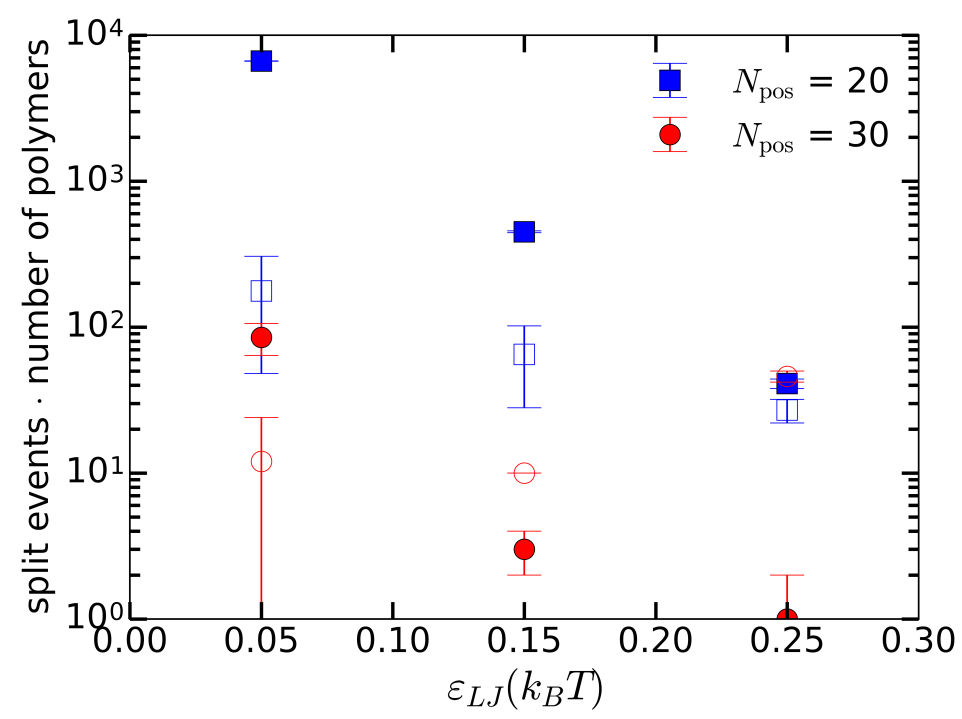

Figure SI 8.1: Number of expulsion events (filled symbols) and fission events (open symbols) corrected for the number of polymers involved per split event in the time range $2 \times 10^{6} \tau-5 \times 10^{6} \tau$, for $N_{\text {pos }}=20, N_{\text {neg }}=20$ and $N_{\text {neu }}=50$ and for $N_{\text {pos }}=30, N_{\text {neg }}=30$ and $N_{\text {neu }}=75$. 


\section{SI 9 Net charge histograms of expelled complexes}
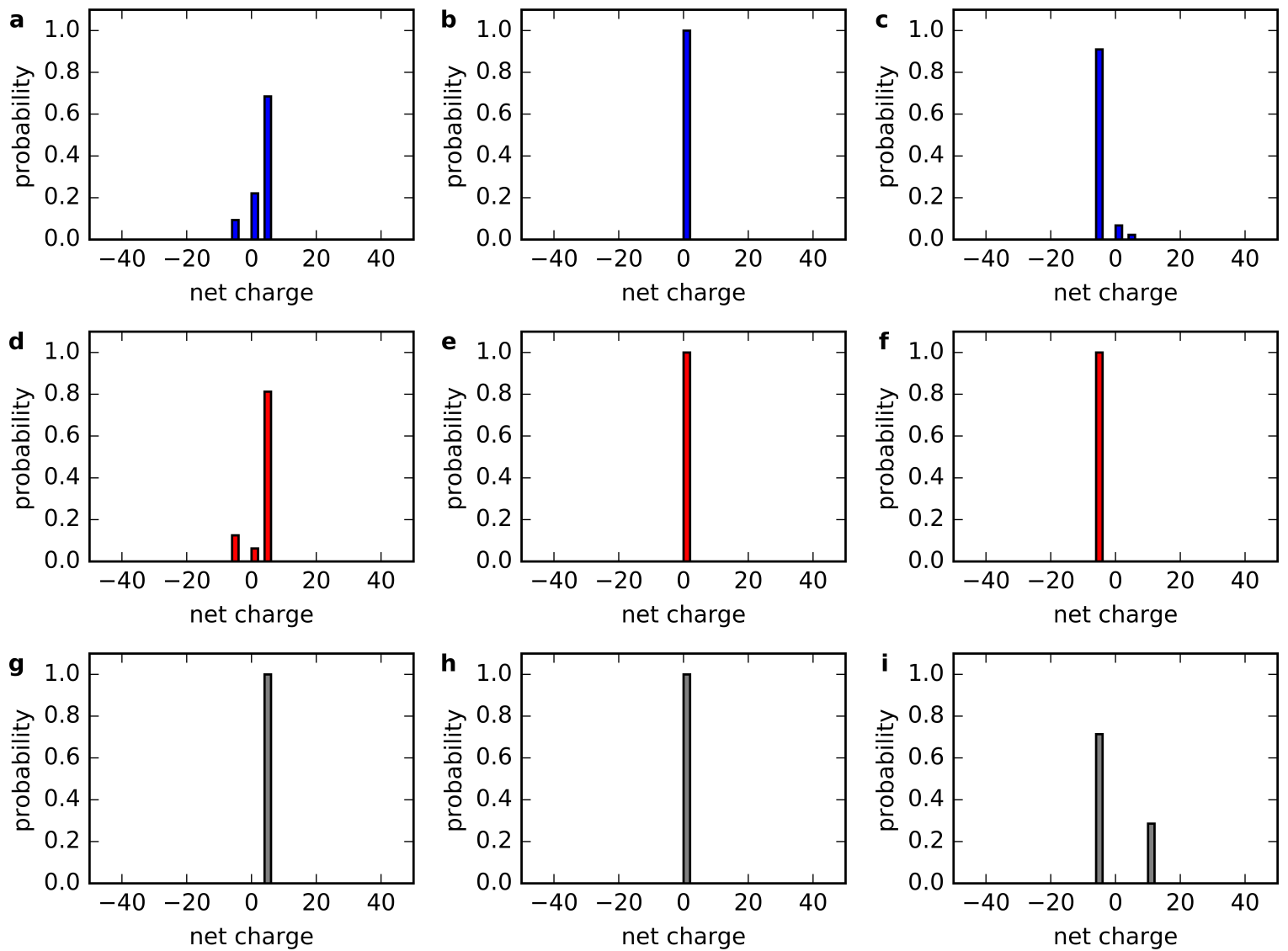

Figure SI 9.1: Histograms of the net charge of the smallest complex formed in a split event for $\varepsilon_{\mathrm{LJ}}=0.05 k_{B} T$ $(\mathrm{a}, \mathrm{b}, \mathrm{c}), \varepsilon_{\mathrm{LJ}}=0.15 k_{B} T(\mathrm{~d}, \mathrm{e}, \mathrm{f})$ and $\varepsilon_{\mathrm{LJ}}=0.25 k_{B} T(\mathrm{~g}, \mathrm{~h}, \mathrm{i})$ and for $N_{\text {neg }}=15(\mathrm{a}, \mathrm{d}, \mathrm{g}), N_{\text {neg }}=20(\mathrm{~b}, \mathrm{e}, \mathrm{h})$ and $N_{\text {neg }}=25$. In all cases, $N_{\text {pos }}=20$ and $N_{\text {neu }}=50$. 